\title{
A Pedagogy-Based Framework for Optimizing Learning Efficiency across Multiple Disciplines in Educational Games
}

\author{
Yunhao Huo
}

\begin{abstract}
Educational games are gaining recognition and popularity as a novel teaching technology in the recent decade. However, most existing studies on its design either fail to examine game mechanics through the lens of pedagogical theories or address the design variation in games integrating different fields of study. This paper aims to resolve the deficiencies in prior research and shed light on the heuristic of creating effective educational games through a structured, pragmatic, and multidimensional design framework. An empirical study is conducted on 50 representative games stratified based on 5 categories of academic disciplines. A total of 25 game mechanics that promote learning outcomes are identified and analyzed in relation to three schools of learning theories-behaviorism, cognitivism, and constructivism. Finally, the findings in game mechanics, academic disciplines, and learning theories are synthesized to derive a pedagogy-based framework with the intent to help game designers optimize learning efficiency in their games and create a better learning experience for players.
\end{abstract}

Index Terms-Game-based learning, educational game, game design, pedagogy.

\section{INTRODUCTION}

Playing has long been proven an integral part of children's development not only as an intrinsically motivated behavior for recreation but also the lens through which children experience and learn about the world [1]. Game-based learning is a pedagogical method widely employed by educators from the past to the present that makes uses of the positive effect of playing to enhance educational outcomes [2] However, historical evidences reveal that such a method seems to be limited to certain disciplines (e.g., mathematical teaching) [3]. The advent of computer technology brings new possibility to the field of game-based learning. With the unprecedented processing power and graphic technology of computers, modern educational games can represent, simulate, and contextualize knowledge from an expansive spectrum of academic disciplines [4]. As video game becomes a ubiquitous form of entertainment that exerts significant influence on contemporary society and culture [2], the prospect and challenge for educational games rise as well. On the one hand, a multitude of studies has proven the positive effect of educational games on players' cognitive

Manuscript received May 13, 2019; revised July 3, 2019. This work was supported in part by the U.S. Department of Commerce under Grant BS123456.

Yunhao Huo is with Bishop O'Dowd High School, Oakland, CA 94605 USA (e-mail: howardhuousa@gmail.com). development and learning outcomes [4]-[12]. On the other hand, educational games that lack manifest recreational value or concentrate too much on the "play" part often fail to attain the educational objective [10], [13].

The potential and design difficulty of educational games call for the institution of a framework that provides pragmatic guidance for optimizing learning outcomes and addresses the design variation across different disciplines in educational games. However, most existing studies focus on a certain aspect of game design or field of study only. Few researchers encompass multiple aspects of design [13]-[17] or discuss the design variation across different games [2], [5], [18], but none of them addresses both issues. This paper aims to address the deficiencies in prior research and offer a comprehensive and pedagogy-based view into the design heuristic of effective educational games. The major difference between the current study and previous studies is that the former incorporates the following three aspects cohesively in the analysis and generalization of game mechanics. First, this study analyzes game design from a pedagogical perspective. The effect of all mechanics identified is examined through the lens of three learning theories to ensure its value in improving learning efficiency. Second, the study explores various knowledge structures across different academic disciplines and specifies the usability of each mechanic proposed in the framework. Third, the paper offers a structured examination of the interrelation between game mechanics, academic disciplines, and pedagogical theories. The findings and proposals in this work are intended to aid game designers in creating and optimizing educational games for better learning outcomes and establish a theoretical basis for further studies.

\section{THEORETICAL FRAMEWORK}

In order to understand the subject difference in game design, we need to examine game-based learning from a pedagogical point of view [18]. Despite numerous learning theories developed throughout the history, they can be categorized into three schools: behaviorism, cognitivism, and constructivism [19], [20]. Behaviorism argues that learning are essentially reactive behaviors to a given stimulus [21], [22]. In game design specifically, behaviorists focus on establishing and reinforcing stimuli-response (S-R) model through repetitive practice as a means to achieve mastery [20], [23]. In contrast, cognitivism shifts the focus to learners' processing and application of new information in organization with existing knowledge [24], [25]. Transferred into game design, this theory stresses the explanatory and 
simulative function of video games; games should facilitate players' higher-level cognitive processes through organized knowledge structure and simulated application scenarios [14, 17, 20]. On the other hand, constructivism, according to Piaget and Vygotsky's theories, postulates that people learn through individualized experience and social interaction, which help learners understand their own reality [26], [27]. Constructivist designs in educational games emphasize the unique and adaptive experience-based learning and collaborative task [18], [28]-[33]. Based upon these three pedagogical theories and their distinct emphasis on learning approach, we propose six categories of game mechanics that exert influence on players' learning experience and educational outcomes (See Fig. 1).

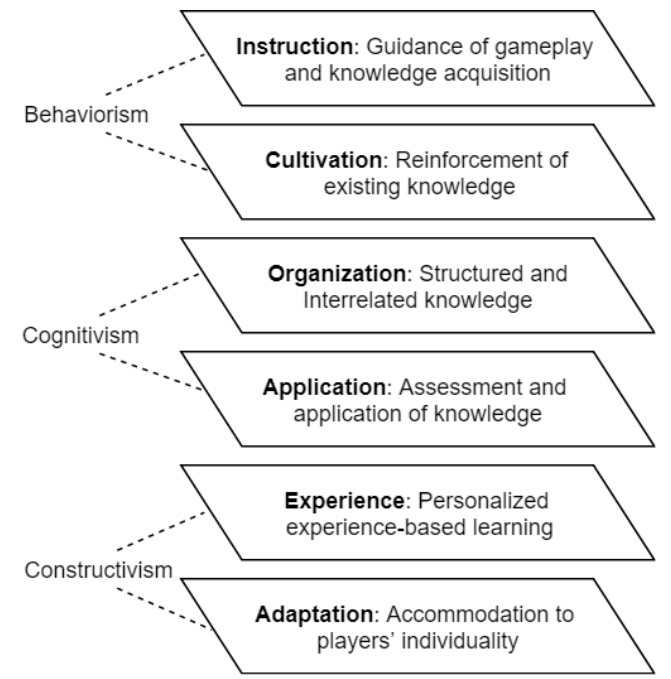

Fig. 1. Six categories of game mechanics based on three schools of learning theories.

As the type of study and the corresponding knowledge structure implemented in different games vary, the most applicable and effective pedagogical theory for each game may vary as well [2], [18]. In order to develop a heuristic that takes into consideration the difference between fields of study, we adopt a conventional classification of academic disciplines and categorize educational games into five types: Natural Science Games (NSG), Formal Science Games (FSG), Social Science Games (SSG), Professional Skill Games (PSG), and Humanities Games (HMG) [34]. The examples of subjects included in each category can be found in Fig. 2 .

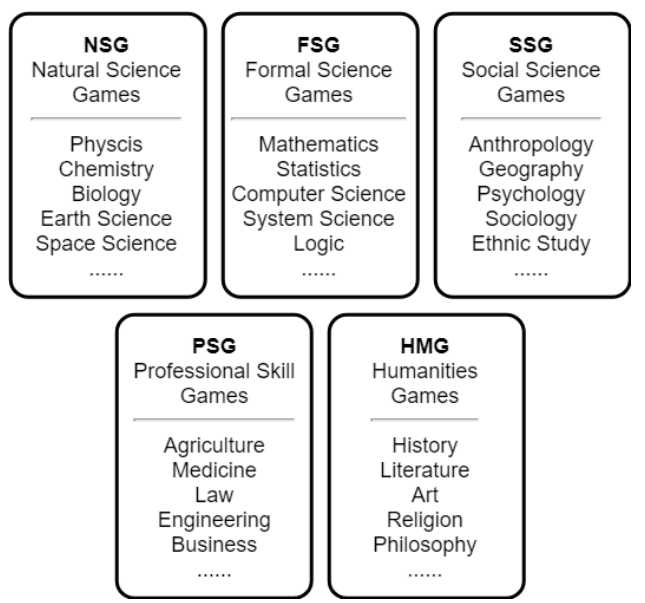

Fig. 2. Classification of educational games based on their academic disciplines.
As we formulate the categories of educational game and mechanics, an in-depth study of the impact of different mechanics and learning theories on different types of subjects integrated in educational games becomes possible.

\section{MECHANICS StUdy}

\section{A. Method}

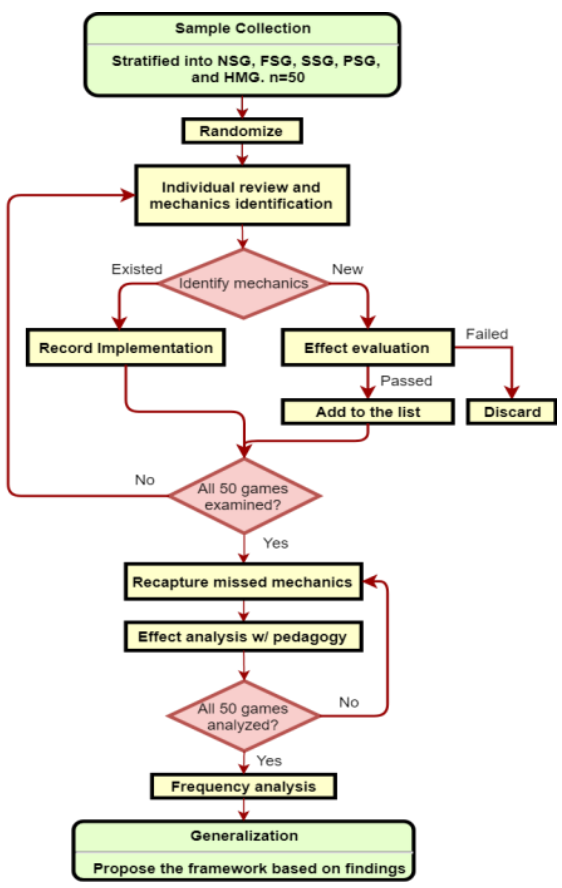

Fig. 3. Flowchart of the mechanics study carried out.

TABLE I: THE FREQUENCY OF MECHANICS

\begin{tabular}{|c|c|c|c|}
\hline Mechanics & Category. & Freq. & $\begin{array}{c}\text { Common Game } \\
\text { Type }\end{array}$ \\
\hline Instructional Objective & Instruction & 0.48 & $\begin{array}{l}\text { NSG, PSG } \\
\text { All } \\
\text { NSG, PSG, }\end{array}$ \\
\hline Contextualized Knowledge & Instruction & 0.74 & PSG \\
\hline Multidimensional Material & Instruction & 0.60 & PSG \\
\hline Embedded Teaching & Instruction & 0.46 & PSG \\
\hline In-game Reference & Instruction & 0.52 & All \\
\hline Tips and Hints & Instruction & 0.50 & All \\
\hline Learning-driven Task & Cultivation & 0.72 & NSG, FSG, PSG \\
\hline Iterative Learning & Cultivation & 0.72 & FSG, PSG \\
\hline Corrective Feedback & Cultivation & 0.58 & NSG, SSG \\
\hline Performance Evaluation & Cultivation & 0.80 & NSG, SSG \\
\hline Modular Learning & Organization & 0.52 & NSG, SSG \\
\hline Structured Knowledge & Organization & 0.52 & Except FSG \\
\hline Concept Matching & Organization & 0.34 & All \\
\hline Interrelated Teaching & Organization & 0.64 & SSG, PSG \\
\hline Assessment of Knowledge & Application & 0.78 & All \\
\hline Application Simulation & Application & 0.60 & SSG, PSG, \\
\hline Randomizable Content & Application & 0.68 & HMG \\
\hline Multiple Strategies & Application & 0.60 & SSG, PSG, \\
\hline Educational Narrative & Experience & 0.62 & HMG \\
\hline Immersive Scenario & Experience & 0.56 & SSG, PSG \\
\hline Collaborative Task & Experience & 0.24 & SSG, PSG \\
\hline Explorative Task & Adaptation & 0.56 & NSG, PSG, \\
\hline Adaptive Difficulty & Adaptation & 0.56 & HMG \\
\hline Content Customization & Adaptation & 0.46 & SSG, PSG, \\
\hline \multirow[t]{3}{*}{ Branches of Progression } & Adaptation & 0.54 & HMG \\
\hline & & & PSG \\
\hline & & & All \\
\hline
\end{tabular}




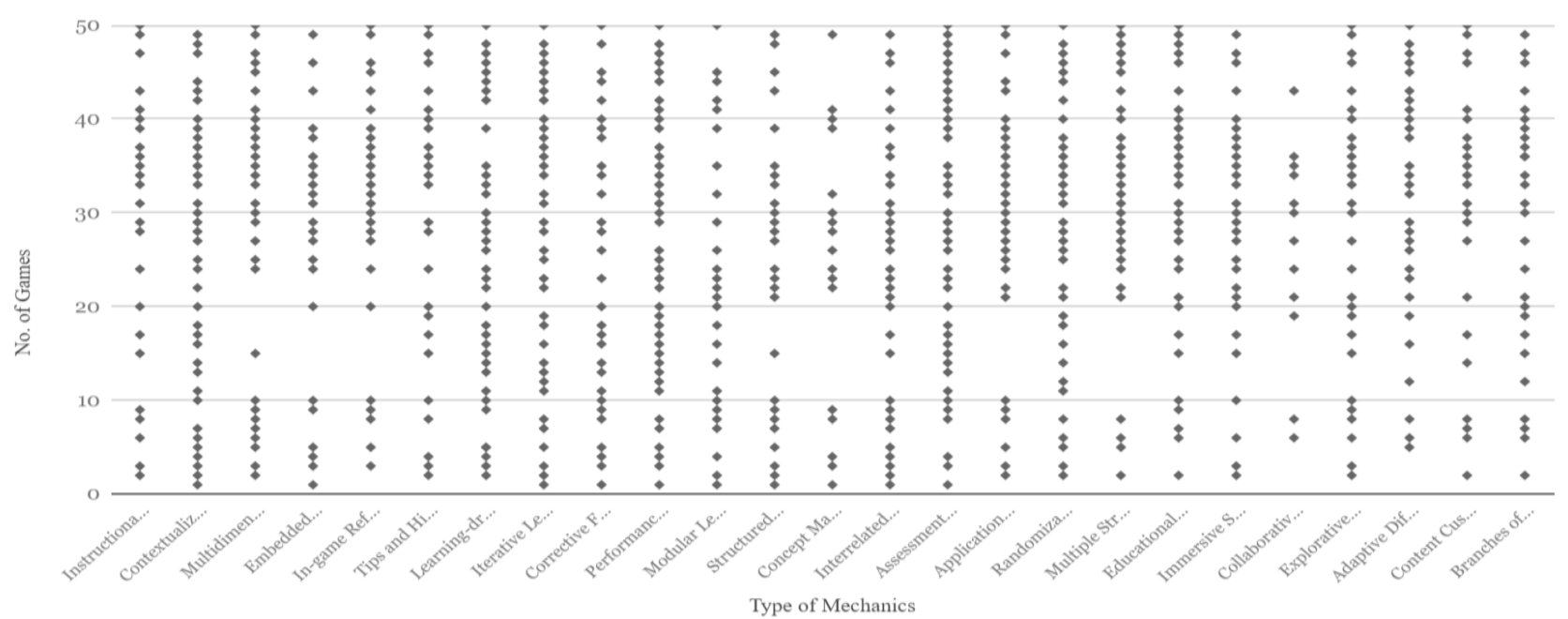

Fig. 4. Plot of the occurrence of each mechanics in 50 games studied. The number of games is sorted based on subject category. No. 1-10 are NSG. No. 11-20 are FSG. No. 21-30 are SSG. No. 31-40 are PSG. No. 41-50 are HMG.

The primary goal of this study is to identify and analyze game mechanics used in different educational games that improve players' learning efficiency and overall learning outcome. Therefore, we collected a sample of 50 representative games and carry out two stages of mechanics study (See Fig. 3). Our sample is stratified based on the five categories of academic disciplines: Natural Science Games, Social Science Games, Formal Science Games, Professional Skill Games, and Humanities Games; each category is comprised of 10 games varying in genres, size, and difficulty. The inclusion criteria are 1) the game demonstrates outstanding popularity in the container site or third-party player forums 2) the game is positively rated on its educational content and effect by users or credible institutions 3 ) the container or developer of the game is reliable and experienced in hosting or creating educational games.

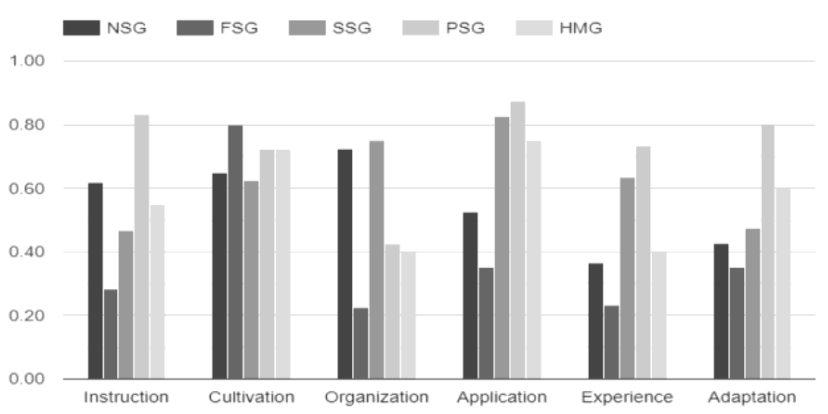

Fig. 5. Comparison of mechanics frequency in five types of games across six mechanics categories.

In the first stage of mechanics study, each selected game undergoes an independent review on its design and mechanics implemented. The order of examination is randomized to avoid bias posed by consecutive homogenous academic disciplines and container sites. For each new mechanic identified, it is evaluated on whether it 1) fosters the development of a specific skill or knowledge 2) contributes to the overall improvement of learning efficiency and outcome. If either of the criteria are met, the mechanic will be included for further analysis and classified into one of the six categories. Eventually, 25 mechanics are selected and categorized (See Table I). In the second stage of mechanics study, each game is reexamined in comparison to the 25 mechanics identified-any mechanics omitted in the previous stage are included. Then, the specific implementation of each mechanic is analyzed in relation to the type of knowledge integrated in the game, its effect on promoting efficient learning, and its corresponding pedagogical theories. The occurrence of each mechanic in the 50 games examined is marked as a dot in Fig. 4.

\section{B. Observation}

As shown in Fig. 4, the distribution of mechanics is drastically uneven across the plot. This imparity is primarily the result of different academic disciplines and their varying inherent knowledge structure (See Fig. 5 for frequency difference of mechanics among subjects). It is found that Natural Science Games have above-average percentage of instructing players' learning and organizing embedded knowledge, but few contexts for the application of players' acquired knowledge. NSG also demonstrates weak integration of experience-based learning and adaptation to players' individuality. Formal Science Games stand out in terms of cultivating players' skill (i.e., through iterative practice), but fall short in other 5 mechanics categories. Social Science Games feature the most organized learning and surpass the mean percentage in knowledge application and experience-based learning. Cultivation design in SSG, however, is the rarest among all game categories. Professional Skill Games encompass the most adaptive and instructional mechanics and excel in providing educational experience and application scenarios, but they have a mediocre percentage of cultivation and organization design. Finally, Humanities Games tend to include adaptive design to match players' knowledge levels and contextualized application of educational gains. Their percentage of instructional mechanics is also higher than the average, but HMG generally lack organization of content and immersive experience.

There are a few exceptions against general trends. First, business games (subordinate to PSG) commonly implement collaborative tasks, which raises the overall percentage of experience-related mechanics of PSG. Nevertheless, most other types of PSG rarely include such a mechanic. Second, despite generally loose integration of instructional design in FSG and HMG, both types of game utilize contextualized 
knowledge to a great extent. Similarly, knowledge assessment and randomizable content are prevalent in FSG whereas other application-related mechanics are mostly absent. Third, although educational narrative and immersive scenarios prevail in HMG, collaborative design is rarely implemented. Consequently, it lowers HMG's overall frequency of Experience-related design compared to SSG and PSG.

\section{Analysis}

In order to shed light on the underlying cause behind the design variation among different types of games, their knowledge structure and characteristics need to be identified and analyzed [12]. Although no definitive method is available for categorizing knowledge, we adopt a widely used classification of knowledge in this paper: logical (causal relation and logic), semantic (definition and meaning), systemic (organized relation between knowledge), and empirical (observation and experience) [35]. All four types of knowledge can be found across all types of educational games, but the degree to which they are integrated and facilitate educational goals varies among academic disciplines [9]. Fig. 6 presents the most prominent types of knowledge integrated in each of the five game categories along with their common types of game mechanics implemented.

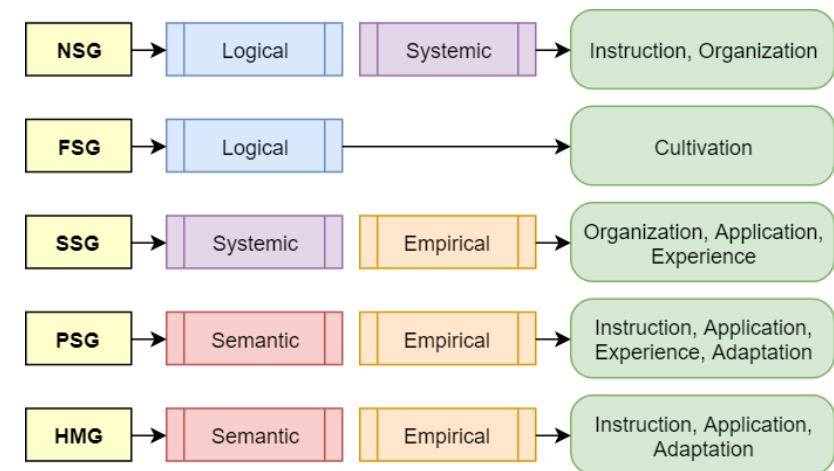

Fig. 6. The most prominent types of knowledge and mechanics in each of 5 game categories.

Most of FSG examined are lighter games comprised of iterative assessment as opposed to observation-based or experience-based design in other types of game. This is essentially due to the abstract and logical knowledge structure as well as the model in which learners improve the corresponding skills in FSG [7]. Behaviorist designs, such as knowledge assessment and corrective feedback, are suitable for the practice-based learning in FSG [15]. Players reinforce their skill through iterative cycles of assessment and feedback, which gradually establish a stimuli-response model for processing abstract information in various scenarios [36]. By utilizing a randomized and adaptive content flow, the coverage of players' S-R model is enlarged, hence achieving a greater proficiency in the tested skill and eventually mastery. In order to reduce tedium and players' resistance to conceptual knowledge, most FSG contextualize learning and assessment in an engaging way (e.g., represent mathematical problems as sports). Motivational design such as trophies, achievements, and players' competitions are also frequently employed in FSG [37].

The learning in NSG and SSG involves observing phenomenon (empirical), theorizing the cause (logical), and contriving rules and laws (systemic) applicable to a greater scope [38]. Both types of game employ organizational design more frequently than others. However, the generalization of knowledge is more mathematical in NSG whereas it is primarily empirical in SSG. As the ramification, NSG employ instruction more effectively and SSG utilize application and experience-based learning to a greater degree. Cognitivist mechanics (e.g., concept matching, education of knowledge interrelation etc.) work in accordance with the structured learning in NSG and SSG [24]. Players learn through fostering higher-level reasoning, information processing, and organization skills [20], all of which facilitate learning activities, particularly result generalization and trend prediction. Thus, they lead to higher educational outcomes in NSG and SSG.

In both PSG and HMG, players often learn through an educational narrative, environment, or simulated scenarios (empirical) [14]. The difference is that the immersion in PSG focuses on skill fosterage whereas in HMG players are more likely to study the meaning and application of an experience (semantic) via observation and meta-reflection. The Constructivist approach is compatible with this type of learning as it allows players to construct their own understanding of knowledge and adapt learning experience to their individuality [30]-[32]. By encouraging players to explore various strategies and branches of progression, such a design sparks their intrinsic motivation and promotes multifaceted educational gains [37]. In PSG, additionally, instruction and assessment are usually embedded into the simulated setting to guide players' acquisition and mastery of intended skills. Certain mechanics, such as collaboration, may augment learning fruition in games that place a focus on specific skills (e.g., teamwork) [36], [38].

\section{PROPOSED FRAMEWORK}

As the type of learning fostered in different academic disciplines varies, games that incorporate different fields of study should have their specified design heuristic to follow. After studying 50 representative educational games across five subject categories and analyzing them through the lens of knowledge structure and learning theories, we propose a pedagogy-based framework that provides pragmatic design guidelines for educational games of different academic disciplines and focuses. The guidelines are specified into three sections; each corresponds to one learning theories. The common applicable subjects are explicitly identified for each mechanic described. Through this multi-perspective design framework, we aim to help game designers optimize the mechanics design in their game based on types of learning integrated and ultimately create an efficient and fruitful learning experience for players.

\section{A. Behaviorism}

1) The in-game objectives should explicitly direct player toward completing learning-related tasks. Common subjects: games with potentially large distraction such as NSG, SSG, and PSG.

2) The knowledge should be represented, simulated, or explained in a contextualized setting. Common 
subjects: all types of games.

3) The game should include a multifaceted presentation of knowledge such as different perspectives, senses, or media. Common subjects: games featuring complex or indefinite knowledge such as SSG, PSG, and HMG.

4) Direct teaching or instruction should be provided for novel or complex knowledge and skills. Common subjects: all types of games.

5) In-game reference such as a glossary should be integrated to assist learning, supplement knowledge, or resolve confusion. Common subjects: games utilizing complex and semantic knowledge such as NSG, SSG, PSG, and HMG.

6) The learning or practice of knowledge and skills should be integrated into in-game tasks and necessary for progression. Common subjects: all types of games.

7) Players should be able to extend learning or improve their skills through iterative assessment-improvement cycles. Common subjects: games that center at skill fosterage such as FSG and PSG.

8) Corrective feedback should be provided when players make learning-related mistakes to effectively guide them toward intended learning outcomes. Common subjects: all types of games.

9) Players' performance should be evaluated and presented periodically to help players adjust their learning pace and goals. Common subjects: all types of games.

B. Cognitivism

1) Learning content should be modularized to promote efficient learning and create a more organized learning structure. Common subjects: games with complex knowledge such as NSG, SSG, and PSG.

2) Knowledge should be purposefully structured (e.g., the concepts that come later are built upon previously taught knowledge) to facilitate players' comprehension. Common subjects: games with complex and interrelated knowledge such as NSG, SSG, and PSG.

3) Knowledge matching mechanics (e.g., direct line-matching or scenarios-based matching) may be utilized to aid comprehension of knowledge interrelation. Common subjects: games featuring a network of knowledge such as NSG and SSG.

4) Interrelation between knowledge should be directly informed or implicitly revealed. Common subjects: games with complex and systemic knowledge such as NSG and SSG.

5) Players' educational gains should be assessed periodically to provide knowledge application, ensure learning effectiveness, and allow improvement. Common subjects: all types of games.

6) Various application scenarios should be integrated for contextualized learning and application of knowledge in multiple situations. Common subjects: multifaceted application-centered games such as SSG and PSG.

7) The setting, learning, and assessments should be randomized to expand players' knowledge coverage, provide additional practice, and enhance replayability. Common subjects: all types of games.
8) The objectives should be attainable via multiple strategies to encourage explorative application of knowledge. Common subjects: experience-based and explorative games such as SSG, PSG, and HMG.

\section{Constructivism}

1) Educational concepts should be embedded into an immersive narrative to contextualize knowledge and allow players' diversified understanding of it. Common subjects: experience-based games such as SSG and PSG.

2) The application scenarios should be immersive and simulated based on real-life situation. Common subjects: skill-fosterage games such as SSG and PSG.

3) Multiplayer mechanics may be integrated to cultivate collaborative skills or simulate a realistic environment (e.g., trading market). Common subjects: games with a specific emphasis on social skills or realistic simulation, such as business games and management games [38]. Multiplayer competition may also be used to motivate learning in all types of games [39].

4) Players should be able to explore the story, environment, and mechanics under their own preference and curiosity. Common subjects: observation-based and experience-based games such as NSG, SSG, and PSG.

5) Multiple difficulty levels should be provided and adjusted according to players' performance (e.g., suggest a lower difficulty if players are stuck at one level for too long). Common subjects: all types of games.

6) Players should be able to customize the game's content, preference, and in-game characters for personalized learning experience. Common subjects: games that center at individual experience such as PSG.

7) Multiple branches of progression (e.g., storylines, objectives) should be designed for players to choose based on their own perspective and preference. This design help players construct individualized understanding of knowledge in games that foster personal skills development such as PSG.

\section{CONCLUSION}

In this paper, we examine the design of 50 educational games from 5 subject categories and analyze 25 integrated game mechanics in relation to behaviorism, cognitivism, and constructivism. Our findings indicate that behaviorist design such as instruction and cultivation are commonly used in games with a focus on logical and semantic knowledge. Cognitivist design such as organization and application appear most frequently on games whose knowledge structure is systemic. Constructivist design such as experience and adaptation favor games to which empirical learning is the most essential. In terms of subject, Formal Science Games are the most common employer of behaviorist design. Natural Science Games and Social Science Games utilize cognitivist approach to a great extent. Professional Skill Games and Humanities Games, on the other hand, implement the most constructivism-based mechanics. By utilizing our proposed 
framework, game designers should be able to select and implement mechanics that are most suitable for the specific subject of their games, hence optimize the learning efficiency and educational outcomes.

\section{CONFLICT OF INTEREST}

The author declares no conflict.

\section{AUTHOR CONTRIBUTIONS}

The author had approved the final version.

\section{REFERENCES}

[1] J. Goldstein, "Play in children's development, health, and well-being," 2012.

[2] M. Dipietro et al., "Towards a framework for understanding electronic educational gaming," Journal of Educational Multimedia and Hypermedia, vol. 16, no. 3, pp. 225-248, 2007.

[3] J. A. Betz, "Computer games: Increase learning in an interactive multidisciplinary environment," Journal of Educational Technology Systems, vol. 24, no. 2, pp. 195-205, 1995.

[4] F. Ke, "Designing and integrating purposeful learning in game play: A systematic review," Educational Technology, Research and Development, vol. 64, no. 2, pp. 219-244, 2016.

[5] D. Vlachopoulos and A. Makri, "The effect of games and simulations on higher education: A systematic literature review," International Journal of Educational Technology in Higher Education, vol. 14, pp. 1-33, 2017.

[6] F. Alonso et al., "Learning objects, learning objectives and learning design," Innovations in Education and Teaching International, vol. 45, no. 4, pp. 389-400, 2008.

[7] P. H. Siew, "Pedagogical change in mathematics learning: Harnessing the power of digital game-based learning," Journal of Educational Technology \& Society, vol. 21, no. 4, pp. 259-276, 2018.

[8] S. M. Loyens and D. Gijbels, "Understanding the effects of constructivist learning environments: Introducing a multi-directional approach," Instructional Science, vol. 36, no. 5-6, pp. 351-357, 2008.

[9] D. B. Clark et al., "Digital games, design, and learning: A systematic review and meta-analysis," Review of Educational Research, vol. 86, no. 1, p. 79, 2016.

[10] W. Westera, "Why and how serious games can become far more effective: Accommodating productive learning experiences, learner motivation and the monitoring of learning gains," Journal of Educational Technology \& Society, vol. 22, no. 1, pp. 59-69, 2019.

[11] N. Ismail and M. Tyler-Jones, "Games-based online course design: Prototype of gamification for online tutors," Reading: Academic Conferences International Limited, 2018.

[12] T. M. Connolly et al., "A systematic literature review of the empirical evidence on computer games and serious games," Computers \& Education, vol. 59, no. 2, pp. 661-686, (2012.

[13] G. Žavcer et al., (2014). Design pattern canvas: An introduction to unified serious game design patterns. Interdisciplinary Description of Complex Systems, 12(4), 280-292.

[14] T. Zarraonandia et al., "Designing educational games through a conceptual model based on rules and scenarios," Multimedia Tools and Applications, vol. 74, no. 13, pp. 4535-4559, 2015.

[15] A. C. McLaren, "Designing effective e-learning: Guidelines for practitioners," Distance Learning, vol. 5, no. 2, pp. 47-57, 2008.

[16] S. P. Walz and S. Deterding, "Gameful world: Approaches, issues, applications," 2015.

[17] C. Hébert and J. Jenson, "Digital game-based pedagogy: Exploring teaching strategies for classroom teachers in the use of video games in K-12 classrooms," Reading: Academic Conferences International Limited, 2017.

[18] C. S. Ang et al., "Linking pedagogical theory of computer games to their usability," International Journal on ELearning, 7(3), pp. 533-558, 2008.

[19] B. B. Lucia and N. M. Rubens, "Learning theory and instructional design using learning objects," Journal of Educational Multimedia and Hypermedia, vol. 13, no. 4, pp. 343-370, 2004.

[20] A. Ahlbrand. (2017). Learning theories and law: Behaviorism, cognitivism, constructivism. [Online]. Available: https://ripslawlibrarian.wordpress.com/2017/03/14/learning-theoriesand-law-behaviorism-cognitivism-constructivism/

[21] J. Moore, "Three views of behaviorism," The Psychological Record, vol. 63, no. 3, pp. 681-691, 2013)

[22] C. Winch, "The philosophy of human learning," 1998.

[23] S. Egenfeldt-Nielsen, "Third generation educational use of computer games," Journal of Educational Multimedia and Hypermedia, vol. 16, no. 3, pp. 263-281, 2007.

[24] K. Yilmaz, "The cognitive perspective on learning: Its theoretical underpinnings and implications for classroom practices," The Clearing House, vol. 84, no. 5, p. 204, 2011.

[25] C. Kirwan, "Making sense of organizational learning: Putting theory into practice," 2013.

[26] B. J. Wadsworth, Piaget's Theory of Cognitive and Affective Development: Foundations of Constructivism, White Plains, NY, England: Longman Publishing, 1996.

[27] C. H. Liu and R. Matthews, "Vygotsky's philosophy: Constructivism and its criticisms examined," International Education Journal, vol. 6, no. 3, pp. 386-399, 2005.

[28] A. H. Almala, "Applying the principles of constructivism to a quality E-learning environment," Distance Learning, vol. 3, no. 1, pp. 33-40, 2006.

[29] J. Mattar, "Constructivism and connectivism in education technology: Active, situated, authentic, experiential, and anchored learning," Revista Iberoamericana De Educación a Distancia, vol. 21, no. 2, pp. 201-217, 2018.

[30] G. Hwang et al., "Development of a personalized educational computer game based on students' learning styles," Educational Technology, Research and Development, vol. 60, no. 4, pp. 623-638, 2012.

[31] E. Andersen, "Optimizing adaptivity in educational games," in Proc. International Conference on the Foundations of Digital Games, 2012.

[32] Y. Karagiorgi and L. Symeou, "Translating constructivism into instructional design: Potential and limitations," Journal of Educational Technology \& Society, vol. 8, no. 1, pp. 17-27, 2005.

[33] W. H. Sultan, P. C. Woods, and A.-C. Koo, "A constructivist approach for digital learning: Malaysian schools case study," Journal of Educational Technology \& Society, vol. 14, no. 4, pp. 149-63, 2011.

[34] A. Oleson, "The organization of knowledge in modern America," 1979.

[35] P. A. Pecorino. Types of knowledge. [Online]. Available: http://www.qcc.cuny.edu/SocialSciences/ppecorino/INTRO_TEXT/C hapter\%205\%20Epistemology/Types_of_knowledge.htm

[36] L. R. Rivers, "The role of computer simulations and digital gaming in distance education," Distance Learning, vol. 14, no. 2, pp. 17-26, 2017.

[37] M. D. Dickey, "Engaging by design: How engagement strategies in popular computer and video games can inform instructional design," Educational Technology, Research and Development, vol. 53, no. 2, pp. 67-83, 2005.

[38] C. Chen et al., "The comparison of solitary and collaborative modes of game-based learning on students' science learning and motivation," Journal of Educational Technology \& Society, vol. 18, no. 2, pp. 237-248, 2015.

[39] C. Chen et al., "How competition in a game-based science learning environment influences students' learning achievement, flow experience, and learning behavioral patterns," Journal of Educational Technology \& Society, vol. 21, no. 2, pp. 164-176, 2018.

Copyright $\odot 2019$ by the authors. This is an open access article distributed under the Creative Commons Attribution License which permits unrestricted use, distribution, and reproduction in any medium, provided the original work is properly cited ( $\underline{\mathrm{CC} B Y} 4.0)$.

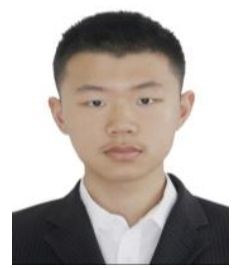

Yunhao Huo was born in Xiamen, Fujian, China. He went to the United States for high school education in 2017. Now he is currently a senior at Bishop O'Dowd High School in Oakland, California, the United States. He started to work as an independent game developer since 2016. He created alter arena in 2017, which entered the game development world championship. His research focus on game mechanics analysis and educational game design. 